\title{
Paul Langevin
}

Paul Langevin (1872-1946) est un physicien précoce et talentueux dont les travaux ont contribué à l'essor de la physique au début du $X X^{e}$ siècle. L'œuvre scientifique de Paul Langevin est féconde et couvre de nombreux domaines, du magnétisme et des gaz ionisés au mouvement brownien et à la relativité.

Nicolas BONOD, nicolas.bonod@fresnel.fr

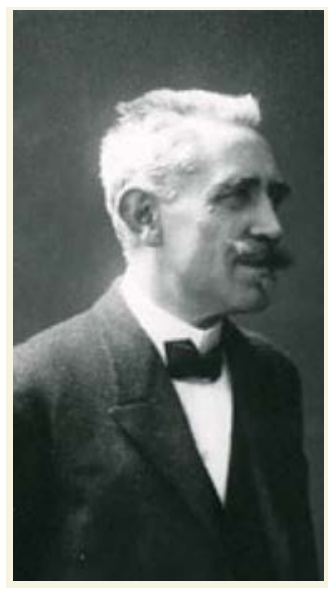

23 janvier 1872 : naissance à Paris

1897

1898

1902

Séjour au laboratoire Cavendish

Thèse de doctorat d'état

Professeur à l'École supérieure de

1905 physique et de chimie industrielles de la ville de Paris

1909 Professeur au Collège de France

19 décembre 1946 : décès à Paris

17 novembre 1948 : transfert des cendres au Panthéon

(C) Henri Manuel, ESPCI-CRH. Image soumise an droit d'auteur et non converte par la licence Creative Commons.

\begin{abstract}
7 omme l'a très justement écrit Louis de Broglie en 1947, "Résumer l'œuvre de Langevin, c'est reprendre $\checkmark$ toute l'bistoire de la physique depuis cinquante ans, et c'est là une tâche difficile sans doute mais d'un passionnant intérêt. " Enseignant réputé et très apprécié de ses élèves, vulgarisateur hors-pair, Paul Langevin s'est aussi pleinement investi dans les causes et luttes qui ont marqué la première moitié du XXe siècle.
\end{abstract}

\section{Enfance et éducation}

Paul Langevin est né le 23 janvier 1872 à Paris dans une famille aux conditions modestes. Son père, Victor-Charles Langevin, métreur-vérificateur dans le bâtiment était républicain et favorable aux Communards. Paul Langevin grandit dans le quartier de Montmartre animé alors par les ouvriers et les artisans. Ses instituteurs détectent rapidement des capacités exceptionnelles pour les matières scientifiques et incitent ses parents à lui offrir des études. Paul Langevin se présente donc à 16 ans au concours de l'école municipale de physique et de chimie industrielles de la ville de Paris qu'il intègre en 1888. Il se distingue dans cette école par ses capacités extraordinaires et en ressort classé au premier rang. Encouragé par son professeur Pierre Curie, il s'oriente vers l'enseignement et la recherche. Il poursuit sa formation scientifique en se présentant en 1893 au concours de l'école normale supérieure de Paris où il est reçu premier. Il intègre l'école en 1894 après son service militaire. Il en ressort diplômé en 1897 et est reçu brillamment cette même année au concours de l'agrégation de physique.

\section{Parcouris académique}

La carrière de chercheur de Paul Langevin va démarrer en 1897 lorsque la ville de Paris lui remet une bourse qui lui permet d'effectuer un séjour scientifique d'une année au laboratoire Cavendish de l'université de Cambridge. Ce laboratoire est un haut lieu de la physique et est alors dirigé par le célèbre physicien Joseph John Thomson. Il rentre à Paris en 1898 et rejoint le laboratoire d'enseignement de la physique de la faculté des sciences de l'université de Paris pour y effectuer sa thèse de doctorat d'état. Il soutient sa thèse intitulée "Recherches sur les gaz ionisés » à l'université de Paris en 1902. Le jury est présidé par Edmond Bouty. Ce sujet a été élaboré durant son séjour au laboratoire Cavendish. Il dédie d'ailleurs son mémoire de thèse au Professeur J.J. Thomson : "Sous l'impulsion donnée par le Professeur J.J. Thomson, l'école des physiciens du Cavendish Laboratory s'est montrée en particulier d'une fécondité surprenante». Le jeune physicien va connaître un début de carrière fulgurant en devenant dès 1902, à l'âge de 30 ans, professeur remplaçant puis suppléant au Collège de France sur la chaire de physique générale et expérimentale d'Éleuthère Mascart. Il devient professeur de physique générale et expérimentale au Collège de France en 1909. En parallèle à ses activités au collège de France, Paul Langevin succède en 1905 à Pierre Curie dont il était le suppléant au poste de professeur à l'École supérieure de physique et de chimie industrielles de la ville de Paris (ESPCI) alors dirigée par Albin Haller. Il y devient directeur des études (1909-1925) puis directeur en 1925, poste qu'il conserve jusqu'à sa mort en 1946 (il sera cependant temporairement démis de ses fonctions durant l'occupation). Ces multiples activités démontrent le fort intérêt que portait Paul Langevin à l'enseignement des sciences.

\section{Contributions scientifiques majeures}

Les activités de recherche de Paul Langevin ont couvert de nombreuses thématiques. Ses principales contributions portent sur les gaz ionisés, le mouvement brownien, la relativité restreinte et les sonars. 
Ses travaux sur les gaz ionisés l'ont amené à étudier la conductivité de l'atmosphère. Il distinguera deux types d'ions: des ions ordinaires obtenus par action des rayonnements sur un gaz, et des ions lourds qu'il nommera « gros ions ». Il réalisera que ces gros ions résultent de la fixation des petits ions sur des particules en suspension dans l'air. Ces travaux auront des répercussions en météorologie sur la compréhension des mécanismes de formation des nuages en haute altitude comme les cirrus, et en basse altitude comme les cumulus et les nimbus.

À la suite de ces premiers travaux, Paul Langevin porte son intérêt sur le développement d'un modèle microscopique du magnétisme. Pierre Curie, son professeur à l'ESPCI, avait montré expérimentalement durant ses travaux de thèse que la susceptibilité magnétique des matériaux paramagnétiques est proportionnelle à l'inverse de la température. Paul Langevin développe un modèle microscopique du magnétisme pour expliquer cette dépendance où chaque atome possède un moment magnétique dû au mouvement des électrons autour du noyau sur une orbite fermée. Il montre que la dépendance en température de la susceptibilité magnétique provient de cette compétition entre les moments magnétiques des atomes qui tendent à s'aligner et l'agitation thermique qui tend à les désordonner. Il utilise alors la loi statistique de Boltzmann pour retrouver une dépendance inversement proportionnelle de la susceptibilité magnétique avec la température.

C'est à l'occasion de ses premiers cours sur la théorie électromagnétique au Collège de France que Paul Langevin s'intéresse aux concepts fondateurs de la relativité : «Bien qu'iln'ait alors rien publié à ce sujet, il semble que Paul Langevin ait aperçu presque en même temps qu'Einstein l'une des conséquences les plus importantes de la nouvelle dynamique : le principe de l'inertie de l'énergie. Ce principe affirme, on le sait, qu'à toute forme d'énergie correspond une masse égale à la valeur de cette énergie divisée par le carré de la vitesse de la lumière dans le vide. » (Louis de Broglie, 1947). Il découvre quelques mois après les travaux publiés par Albert
Einstein sur cette théorie. Il devient alors un ardent promoteur de cette théorie révolutionnaire qu'il enseigne au Collège de France et qu'il diffuse lors de nombreuses conférences. Il propose de mettre en illustration cette théorie par le paradoxe des jumeaux : l'un des jumeaux voyage à une vitesse proche de celle de la lumière alors que l'autre jumeau reste à terre, le temps s'écoulant plus lentement pour le premier que pour le second. Il invite également Albert Einstein à donner plusieurs cours au Collège de France.

Si Paul Langevin n'a pas laissé son nom à ses travaux sur le magnétisme et sur la relativité, son nom restera cependant identifié à ses travaux sur le mouvement brownien. Ce mouvement aléatoire des particules avait été décrit pour la première fois par le botaniste américain Brown pour décrire le mouvement du pollen à la surface de l'eau. Einstein a publié une première théorie en 1905 sur ce mouvement en se basant sur l'étude des chocs entre les molécules du milieu environnant et les particules. Langevin explique ce mouvement en se basant sur l'analyse de la trajectoire d'une particule qui est soumise à une force de frottements et une force résultant de l'ensemble des chocs aléatoires. Il obtient une équation différentielle stochastique, qui est aujourd'hui très utilisée par exemple en biologie pour décrire le mouvement des corps biologiques dans des fluides physiologiques.

\section{Physicien et millitant}

Le parcours de Paul Langevin est exceptionnel de par l'ampleur de ses contributions scientifiques et de ses activités d'enseignement, mais également de par son engagement et son militantisme dans les grands mouvements sociaux de la première moitié du $\mathrm{XX}^{\mathrm{e}}$ siècle. Paul Langevin est né juste après les évènements de la commune de Paris du printemps 1871. "Le fait que j'ai cru devoir partager mes forces entre le service de la Science et celui de la Justice tient certainement

\section{Fast Multi-Channel Photonics Alignment System}

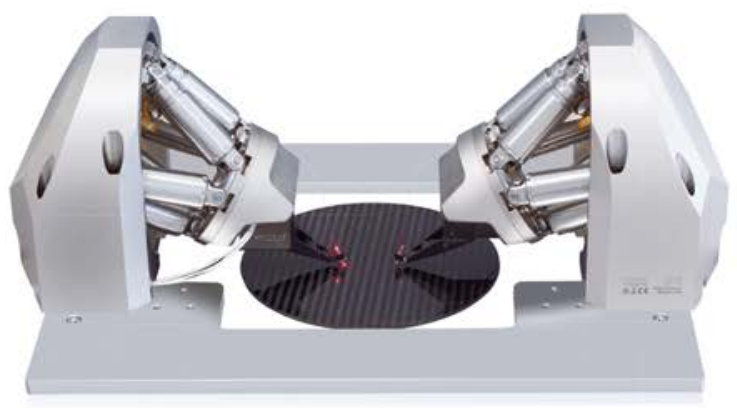

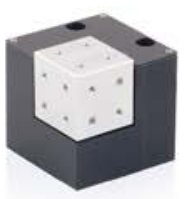

NanoCube

piezo-based

alignment engine

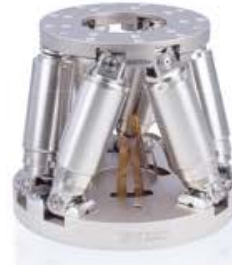

Fast and high-precision

miniature

hexapod 
à l'atmosphère dans laquelle j'aigrandi, au lendemain de la guerre de 1870, entre un père républicain jusqu'au fond de l'âme et une mère dévonée jusqu' au sacrifice, au milieu de cet admirable peuple de Paris dont je me suis toujours senti si profondément solidaire » (3 mars 1945). Son premier engagement public est pris en 1898 alors qu'il est à Cambridge, en signant une pétition en faveur de Dreyfus envoyée par Charles Peguy, son ancien camarade de l'école normale. Paul Langevin adhère à la suite de ce premier engagement public à la Ligue des droits de l'Homme. Durant la première guerre mondiale, il est affecté de 1916 à 1918 au bureau des inventions et y met ses compétences scientifiques au service de la défense du pays. Il développe en particulier un prototype de sonar pour la détection de sous-marins. Son engagement s'accentue à partir de 1920 avec plusieurs prises de position, notamment contre la répression du gouvernement à l'encontre des cheminots grévistes, pour l'amnistie des marins de la mer noire ou encore pour la libération d'André Marty. Il œuvre activement au rapprochement avec l'Allemagne d'après-guerre. Il manifeste aux côtés d'Einstein pour la paix à Berlin en 1923 et fait ouvrir pour lui une chaire au Collège de France (1933). Son engagement s'accentue avec la montée du nazisme dans les années 1930. Il intègre plusieurs comités de congrès, en particulier le congrès mondial de tous les partis contre la guerre impérialiste (Amsterdam, 1932), le « comité de préparation du congrès antifasciste européen » (Paris, 1933) et le « comité mondial de lutte contre la guerre impérialiste » (Amsterdam, 1932). Il devient président du comité mondial de lutte contre la guerre et le fascisme. Il patronne avec le philosophe Alain et l'ethnologue Pierre Rivet le Comité de Vigilance des Intellectuels Antifascistes (CVIA) créé en 1934 et dissout en 1938 à la suite des accords de Munich. Ces trois personnalités signent le 5 mars 1934 le manifeste « Aux travailleurs ». Paul Langevin quitte la direction de ce comité en 1936 à la suite de désaccords entre antifascistes et pacifistes. Ses activités de militant conduisent à son arrestation par les allemands le 30 octobre 1940 dans son bureau de l'école de physique et de chimie. Paul Langevin est détenu à la santé et subit un interrogatoire poussé le 25 novembre. Cette arrestation déclenche un mouvement de protestations et de manifestations dans le milieu universitaire. Face à cette pression, les allemands le libèrent pour le placer en résidence surveillée à Troyes. Il est alors démis de ses différentes fonctions par le gouvernement de Vichy et mis à la retraite en 1941. La répression s'accentue alors. Son gendre et sa fille, tous deux membres du parti communiste, sont arrêtés. Son gendre est fusillé et sa fille est déportée à Auschwitz (elle survivra). Sous la pression de ses amis, Paul Langevin, alors âgé de 72 ans, fuit vers la Suisse en franchissant à pieds la frontière du Jura en mai 1944. Il rentre en France et est accueilli à Annecy en septembre 1944. Il adhère alors au parti communiste et retrouve ses fonctions de directeur de l'école de physique et de chimie en octobre 1944 et de professeur au collège de France. Il préside la «Commission ministérielle pour la réforme de l'enseignement » créée en novembre 1944, plus connue sous le nom de commission Langevin-Wallon.

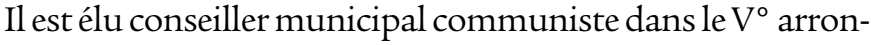
dissement de Paris en mars 1945 . Son $73^{\mathrm{e}}$ anniversaire est l'occasion de lui rendre un hommage solennel dans le grand amphithéâtre de la Sorbonne. Paul Langevin y proclame un discours durant lequel il affirme son soutien au parti communiste. Il devient en 1945 président de la ligue française des droits de l'homme. Il meurt le 16 décembre 1946 dans son appartement de l'école de physique et de chimie. Le journal l'Humanité publie le lendemain un hommage au physicien et au militant. Le gouvernement lui rend un hommage national. Les obsèques ont lieu le 21 décembre 1946. Les cendres de Jean Perrin et Paul Langevin sont transférées au Panthéon le 17 novembre 1948.

\section{POUR EN SAVOIR PLUS}

[1] Louis de Broglie, Notice sur la vie et l'œuvre de Paul Langevin, 15 décembre 1947. Institut de France, Académie des sciences.

[2] Michel Paty, « Paul Langevin (1872-1946), la relativité et les quanta ». Bulletin de la Société Française de Physique, 15-20, 1999

[3] Cécile Michaud, « Les multiples vies de Paul Langevin ». Pourla Science 335, 1999

[4]J. Bok, C. Kounelis, « Paul Langevin (1872-1946). De la butte Montmartre au Panthéon: parcours d'un physicien d'exception ». Reflets de laphysique 1, 14-16, 2006

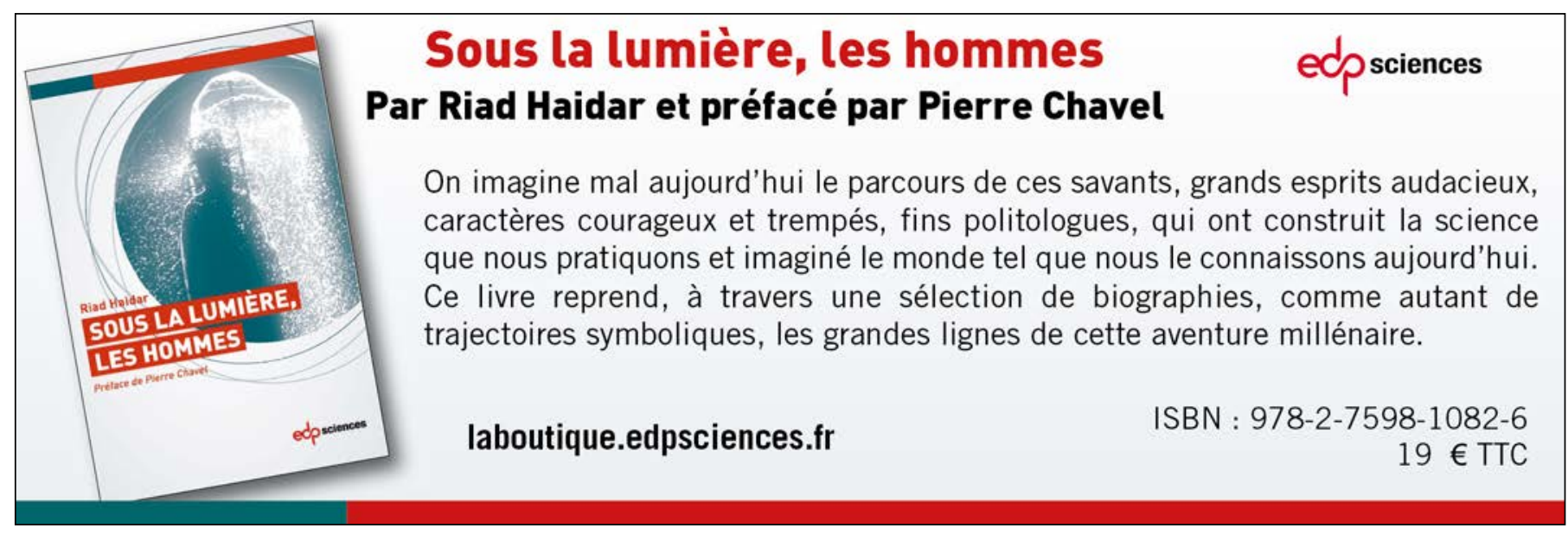

